\title{
Dissociation of early and late face-related processes in Autism Spectrum Disorder and Williams syndrome
}

\section{Alice Gomez ( $\square$ alice.gomez@univ-lyon1.fr)}

Claude Bernard University Lyon 1

\section{Guillaume Lio}

Reference Center for Rare Diseases with psychiatric phenotype Génopsy, le Vinatier Hospital

\section{Manuela Costa}

Technical University of Madrid

\section{Angela Sirigu}

Institut des Sciences Cognitives Marc Jeannerod, Centre National de la Recherche Scientifique

\section{Caroline Demily}

Reference Center for Rare Diseases with psychiatric phenotype Génopsy, le Vinatier Hospital

\section{Research Article}

Keywords: Eye sensitive, Facial features, Fusiform face area, Social brain, Superior Temporal Sulcus

Posted Date: May 7th, 2021

DOl: https://doi.org/10.21203/rs.3.rs-480389/v1

License: (c) (i) This work is licensed under a Creative Commons Attribution 4.0 International License. Read Full License 


\section{Abstract}

Williams syndrome (WS) and Autism Spectrum Disorders (ASD) are psychiatric conditions associated with atypical but opposite face-to-face interactions patterns: WS patients overly stare at others, ASD individuals escape eye contact. Whether these behaviors result from dissociable visual processes within the occipito-temporal pathways is unknown. Using high-density electroencephalography, multivariate pattern classification and group blind source separation, we searched for face-related neural signals that could best discriminate WS $(N=14)$, ASD $(N=14)$ and neurotypical populations $(N=14)$. We found two peaks in neurotypical participants: the first at $170 \mathrm{~ms}$, an early signal known to be implicated in low-level face features, the second at $260 \mathrm{~ms}$, a late component implicated in decoding salient face social cues. The late $260 \mathrm{~ms}$ signal varied as a function of the distance of the eyes in the face stimulus with respect to the viewers' fovea, meaning that it was strongest when the eyes were projected on the fovea and weakest when projected in the retinal periphery. Remarkably, both components were found distinctly impaired and preserved in WS and ASD. In WS, we could weakly decode the $170 \mathrm{~ms}$ signal probably due to their relatively poor ability to process faces' morphology while the late $260 \mathrm{~ms}$ component shown to be eye sensitive was highly significant. The reverse pattern was observed in ASD participants who showed neurotypical like early $170 \mathrm{~ms}$ evoked activity but impaired late evoked $260 \mathrm{~ms}$ signal. Our study reveals a dissociation between WS and ASD patients and point at different neural origins for their social impairments.

\section{Introduction}

Although Autistic Spectrum Disorder (ASD) and Williams syndrome (WS) are both complex developmental conditions with core behavioral deficits in social cognition and face processing, the nature of these deficits differs greatly. Whereas patients with ASD struggle with social interaction and making eye contact, patients with WS seek to do both.

ASD is a neuro-developmental disorder characterized by deficits in social communication and social reciprocity, and repetitive and stereotyped behaviors. Despite extensive research into the biological factors underlying its pathology, behavioral observations remain the principal method of diagnosis ${ }^{2}$. Social deficits, most notably a failure to attend preferentially to the eyes of others, are signs of autism that are observable as early as the first months of life ${ }^{3,4}$.

WS is a rare neurodevelopmental disorder caused by a hemizygous deletion of approximately 25 genes on the 7q11.23 chromosomal region ${ }^{5}$ resulting in a phenotype comprised of medical, cognitive, affective, and neurophysiological impairments ${ }^{6}$. A core behavioral component of this syndrome is increased motivation for social interaction with an apparent lack of fear of strangers ${ }^{7,8}$. Patients with WS have been described as acting as if "everybody in the world is their friend" 8 and their appetitive drive for social interaction and social closeness with other people is commonly likened to hypersociability ${ }^{9}$. 
Patients with WS display atypical facial processing behaviors which are clearly distinct from those of patients with ASD. Specifically, during social interaction, patients with WS appear to exhibit "face fascination" 10 from infancy ${ }^{11}$. Relative to more general forms of visuo-spatial processing, WS patients demonstrate comparative strengths in face processing ${ }^{12,13}$. However, studies have also noted atypical processing in WS patients of eye and mouth regions of the face. Overall, face scanning patterns of individuals with WS differ from those produced by ASD patients. Patients with WS show an increased preference for eyes over the mouth region ${ }^{14,15}$ in upright faces ${ }^{16}$.

In the present study, using the face perception task developed by Lio et al. ${ }^{1}$, we investigated whether a neural signature can be identified that reflects the behavioral dissociation between the two neurodevelopmental disorders.

Faces are multidimensional visual stimuli offering a rich variety of information to observers ${ }^{17}$. The multidimensional nature of this information is key to social interaction. Faces not only convey permanent and stable information such as gender (male or female), race (e.g., Chinese or Caucasian), identity (e.g., John or Mary), but also dynamic and transient information such as emotional expression, direction of attention, and intention ${ }^{18}$.

The neurobiological substrate of these complex cognitive processes relies on at least three brain structures: the occipital and fusiform face areas and the posterior superior temporal sulcus (STS). Studies using PET-scan and fMRI techniques have showed convincing evidence that blood flow increased in the fusiform gyrus in responses to human faces compared to other stimulus viewing, thus leading to its labelling as the fusiform face area (FFA) ${ }^{19-21}$. Techniques with high temporal definition such as EEG have also revealed face-specific evoked potentials (ERPs) in the occipito-temporal cortex. Most notably the N170, a negative potential that peaks at 170 msec is elicited by faces ${ }^{22}$ and by face components, especially the eyes ${ }^{23}$. Furthermore, evidence also suggests that downstream visual regions, such as the occipital face area, a relatively neglected region in face processing, may play a role in coding critical faceparts components ${ }^{24,25}$ during the early stages of processing ${ }^{26,27}$. Studies have so far demonstrated that bold activity in occipital face area (OFA) correlates with ERP face-selectivity at $100-110 \mathrm{~ms}$ after stimulus onset while bold activity in FFA with ERP face-selectivity at $170 \mathrm{~ms}^{26}$. Hence, parts of the face are first processed in the OFA and then computed holistically in the FFA ${ }^{28}$.

Along the occipito-temporal visual stream the STS plays a key role in the human face-perception system, but it is also one of the key components of the 'social brain'. Indeed, brain regions in and around the superior temporal sulcus of both hemispheres may be involved in the analysis of actual or implied facial movements and related cues that provide socially relevant information, such as emotional expression ${ }^{29-}$ 32 and gaze direction ${ }^{33}$. Although establishing which cortical areas generate the N170 is problematic owing to source localization issues ${ }^{34}$, electrophysiological studies suggest that the STS might be crucially involved in generating this ERP in response to face stimuli 35 . Studies that have attempted to 
localize the $\mathrm{N} 170$ to face-selective regions suggest that the N170 reflects neural activity arising from the FFA $^{36}$, pSTS $^{37}$, or both the FFA and pSTS ${ }^{26}$, but not the OFA.

The superior temporal sulcus has also been hypothesized as a key structure associated with the socialinteraction deficits that are typical in patients with in ASD ${ }^{38-40}$. Specifically, this hypothesis points to the STS as playing a role in the early stages of visual processing analysis of social cues. A link between perceptual abnormalities and specific disturbances in social cognition associated with in autism has been proposed. Anatomical and functional alterations in the STS region of ASD individuals corroborate this hypothesis. PET-scan studies have described localized bilateral temporal hypoperfusion in children with autism ${ }^{41}$ as well as a decrease of grey matter and an elongation of the superior temporal sulcus 42,43. In a high-density EEG study while participants performed a face perception task, Lio and colleagues 1 recently showed that evoked activity in the STS occurring at $240 \mathrm{~ms}$ after stimulus onset was significantly high in neurotypical subjects but weak in ASD patients. Furthermore, using multivariate pattern classification analysis, these authors were able to decode and classify brain activity for each group with a high degree of accuracy $(>80 \%)$. This suggests that STS activity is crucial for coding the social value of faces. To measure possible modulation of the STS signal for face subcomponents (eyes, mouth, eyebrows, cheeks etc), Lio et al', in a second task, constrained subjects' attention by presenting each face region in the foveal field. With this design they showed that, in the neurotypical population, the evoked activity is eyes sensitive, that is, the neural signal in the STS source is higher when subjects focused attention on the eyes. In contrast, such modulation was not found in patients with ASD, where evoked activity is not further enhanced when perceiving the eyes. These findings thus demonstrate that in the neurotypical population the $240 \mathrm{~ms}$ evoked response in the STS is linked to the social meaning of faces and more specifically sensitive to eye gaze. Such activity seems missing in the ASD population.

Although, from the perspective of social cognition and face processing at least, ASD and WS conditions appear to reflect two sides of the same coin, the neural substrates that have been proposed as involved in WS diverge from those thought to be implicated in ASD. The regions mainly associated with motivational value (amygdala) and executive control (frontal lobe) have been postulated to play a role in the observed cognitive abnormalities characteristic of WS. It has been hypothesized that maladaptive social behavior in WS is due to lack of inhibition and impaired emotional processing consequent to frontal 44 and amygdala dysfunction ${ }^{45-47}$.

Other studies, however, have suggested that the strong tendency demonstrated by WS patients to approach others and, in particular, to attend to faces ${ }^{48}$ could be explained by additional dysfunctions in another set of neural substrates involved in visual processing. Indeed, WS patients show abnormalities in both the FFA and STS. The FFA has been found to be enlarged ${ }^{49}$ as well as structurally altered ${ }^{45}$ in these patients. Moreover, although WS patients show an overall reduction in brain volume ${ }^{50-52}$, cortical thickness of the superior temporal gyrus has been found increased compared to the neurotypical population ${ }^{53}$. Also, a recent fMRI study using a facial emotion matching task, has shown greater 
activation of the superior temporal sulcus in WS patients compared to patients with anxiety disorders and less activation in the occipital face area compared to controls ${ }^{54}$.

Electrophysiological studies also demonstrated that binding of face features in WS is atypical ${ }^{55}$. In an ERP face recognition task, contrary to controls, who showed an N320 signal for upright face recognition and a P600 for inverted faces, WS patients produced a similar match-mismatch effect on N320 for both upright and inverted faces ${ }^{56}$. In this same study, WS patients showed an abnormal increase in N170 amplitude when viewing trustworthy faces, suggesting that WS patients' hypersociability, may be linked to such enhanced early visual brain activity. In accordance with this idea, Shore and colleagues ${ }^{57}$ have proposed that impairments in low-level perceptual processes might have cascading effects on social cognition.

To identify if the opposite nature of face-related behaviors observed in WS and ASD can also be observed at the neural level, we used the behavioral procedure (see Fig. 1A) designed by Lio et al ${ }^{1}$ that combines a face gender task with high density EEG recording. In this task, we could control the face area focus on by subjects and link it to the cortical response. We examined the EEG brain activity of WS patients compared with data from a group of neurotypical subjects and a group of patients with ASD previously reported by Lio et al. ${ }^{1}$.

Two sets of EEG analysis were performed. First, in a spatio-temporal a priori analysis we built a spatial filter from the STS source identified at $240 \mathrm{~ms}$ by Lio et al. ${ }^{1}$ and used it to extract evoked single-trial activity. Then, for each participant, a map was generated indicating the level of evoked activity as a function of each viewed face area. We expected that, unlike what was found in ASD patients by Lio et al.

${ }^{1}$, face parts that carry a rich amount of social information, such as the eyes, will yield selective STS responses in WS patients, just as was found.

Second, we performed a spatio-temporal a priori analysis. Using a "facial cue" map as regressor of evoked activity, we investigated how much the multichannel EEG signal was able to decode this predicted pattern of evoked activity at every time point. This analysis has the capacity to indicate at which point in time socially relevant facial features of the regressor induce cortical reactivity. Because this method of EEG analysis requires no assumptions regarding cortical source, its use offers a significant advantage over more traditional alternatives, especially when applied to neurodevelopmental disorders.

\section{Materials And Methods}

\section{Participants}

We recruited 14 neurotypical participants ( 5 men and 9 women, mean age $=11.6$, range $=6-21$ ) and 14 WS patients ( 5 men and 9 women, mean age $=11.6$, range $=6-21$ ) from 2018-2019 matched for age and gender. The number of participants in each group was selected to provide a balanced designed across groups, therefore we matched the size of groups to that acquired by Lio et al ${ }^{1}$. We compared the present 
dataset with the EEG data of 14 ASD patients (14 men, mean age $=20$, range $=18-21$ ) recruited in a previous study by Lio et al. ( ${ }^{1}$, Exp. 3). All participants had normal or corrected to normal vision and had no history of psychiatric or neurological disease.

Participants were recruited through national advertisements from WS associations and from the Reference for Rare Diseases GenoPsy Center (Vinatier hospital, Lyon). Each patient received a diagnosis of WS following genetic assessment (deletion at 7q11.23) by the psychiatrist (C.D) involved in the study. Patients with Williams syndrome and neurotypical subjects both participated in a short neuropsychological evaluation to assess visuospatial reasoning, logical thinking and verbal skills (matrix and similarities subtest, WISC-V ${ }^{37}$ ) and visuospatial and auditory attention (Arrows and auditory attention subtest, NEPSY, ${ }^{38}$ ). Unsurprisingly, patients with WS showed lower performances compared to controls in all of these tests (see Table 1). Neuropsychological data of ASD patients obtained from Lio et al.'s study involved IQ evaluation (WAIS IV or WAIS V). Patients with ASD showed normal intellectual abilities (Mean IQ=97.20, SD=27) with the exception of two patients showing lower scores on these tests $(\mathrm{IQ}<75)$.

The study was approved by the French Sud-Ouest Lyon Bérard ethical committee (project $\mathrm{N}^{\circ} 2018$ A02037-48). All methods were carried out in accordance with relevant guidelines and regulations. Prior inclusion, all participants and/or their legal representative provided written informed consent to participate in the study.

\section{Procedure}

Participants were instructed to focus on a fixation cross (with a duration jittered $800 \pm 100 \mathrm{~ms}$ ), which was followed by a face stimulus masked by a Gaussian apodization window (a Full Width at Half Maximum $(F W H M)=10^{\circ}$ ) centered on the fixation cross that appeared for $167 \mathrm{~ms}$ (Figure 1A). Using this procedure, we were able to control which face region subjects were focusing on and the luminance distribution projected on to the retina. To maintain subjects' attention, every $7( \pm 3)$ trial would take the form of a question mark which was presented on the screen instead of a face stimulus. Upon presentation of these trials, participants were required to recall the gender of the last face displayed and indicate it with a button press using either their index or middle finger. Experimental testing was made up of three sessions each consisting of 500 trials. This was preceded by a training session with 100 additional trials to ensure that the gender distinction task was performed above chance-level and that all participants understood the instructions. We set out to control the area of the face focused on by the participant in order to link it to cortical reactivity occurring at 200-300ms. Furthermore, we reduced the number of trials presented to participants to ensure that the duration of task performance did not exceed an hour. This ensured that the task would be more amenable to the attentional abilities of young participants and patients with Williams Syndrome. Following pilot testing with adult participants with WS, we selected a total of 1600 face presentation trials. 


\section{Stimuli}

Face stimuli were identical to those used by Lio et al. (1, Exp. 3). The overall area of each studied face was $20^{\circ}$ in height and $16^{\circ}$ in width. In order to optimize the statistical power of the analysis given the reduced number of trials (1500), the region of focus for each stimulus presentation was no longer randomly selected from all of the pixels in this region. Instead regions were centered on one out of 25 rectangles defined as ROIs. These ROIs were generated by dividing the overall area of the studied face stimuli $\left(20^{\circ}\right.$ height, $16^{\circ}$ width) into a grid of $5 \times 5$, each ROI took the form of a rectangle (size, height $=4^{\circ} \mathrm{x}$ width $=3.2^{\circ}$ ). Given that we presented and recorded 1500 trials, the sampling density of face stimuli was $1500 / 25=60$ trials / ROI / participant. To control the luminance distribution of visual stimuli, despite the different locations of face pictures on the screen, each stimulus was multiplied by a 2-dimensional Gaussian apodization function centered on the fixation cross. The width of the aperture window was chosen so that it was large enough (Full Widths at Half Maximum $=10^{\circ}$ of visual angle) to make the recognition of gender or the identity of each stimulus easy across all trials (Figure $1 \mathrm{~A}$ ).

\section{EEG recording and preprocessing}

We used the Brain Product ${ }^{\text {TM }}$ actiCHamp system to record the electroencephalographic signal from 128 active electrodes (actiCAP 128Ch Standard-2) mounted in an elastic cap at 10-10 and 10-5 system standard locations ${ }^{60}$. All electrode impedances were kept below $50 \mathrm{kOhms}$. Subjects were seated in a darkened, shielded room with their head position controlled by an ophthalmic chin-rest device so that eyelevel was aligned with the fixation cross. EEG data were recorded at a sampling rate of $5000 \mathrm{~Hz}$ with an online reference at the Fz electrode. Offline, data were band pass filtered using zero-phase Chebychev type II filters (Low pass - cutting frequency: $45 \mathrm{~Hz}$, transition band width: $2 \mathrm{~Hz}$, attenuation: $80 \mathrm{~dB}$; order: 35, sections: 18 | High pass - cutting frequency: $0.3 \mathrm{~Hz}$, transition band width: $0.2 \mathrm{~Hz}$, attenuation: $80 \mathrm{~dB}$; order: 9, sections: 5) and re-referenced to a common average. Next, data were epoched from $200 \mathrm{~ms}$ before to $400 \mathrm{~ms}$ after the stimulus onset.

\section{Analysis 1: Spatio-temporal a priori}

First, a spatial filter allowed the extraction of single-trial activity evoked between $200-300 \mathrm{~ms}$ in the STS (Figure 1B). This filter relies on the scalp topography of the source as established by Lio et al. ${ }^{1}$. Then, for each participant, a map was estimated of the level of evoked activity as a function of the focused face area. Finally, for each group, an average was generated that estimated which face area evoked the most activity.

\section{Single trial spatial filtering}

In order to measure the single trial behavior of the component identified in the first experiment by Lio et al.1, we calculated a spatial filter for each trial using minimum variance beamformer techniques 61,62 in 
combination with spatial information estimated at the group level using gBSS (group Blind Source Separation), as described elsewhere 63. For more details, please refer to Experiment 2 of Lio et al. 1.

\section{Gaussian Kernel Density Mapping}

For each subject, we built a source spatial sensitivity map using Gaussian kernel density mapping. This is a non-parametric method that estimates the probability density functions of a random variable. It was weighted here by the intensity of the evoked activity estimated at the source level. This is a similar method to the 'heat map' representations used in eye-tracking studies (see e.g. ${ }^{64}$ ). At each trial $t$, where the focused area was located at the coordinates $\left[x_{t}, y_{t}\right]$ within one of the 25 ROls on face stimuli, we multiplied the mean evoked potential $m_{t}$ extracted from the cortical source of interest, during the previously identified time-cluster [200ms, $300 \mathrm{~ms}$ ] by a two dimensional Gaussian kernel function with a mean value of $\left[x_{t}, y_{t}\right]$ and a Full Width at Half Maximum (FWHM) of $10^{\circ}$ of visual angle. Then, we built the subject-level source spatial sensitivity map by averaging all Gaussian kernel functions. Finally, to highlight the 'most positive' and the 'most negative' areas, the mean value of the map was removed.

We obtained the spatial sensitivity map of the investigated electrophysiological source by performing all signal and statistical analyses on the 25 ROIs ( 25 non-parametric, one tailed, sign tests, $p<0.05$, FWER controlled using the maxT/minP multiple testing procedure) ${ }^{65}$. For visualization purposes only, in order to highlight the face areas that evoked the 'most negative' activities at the subject level, we thresholded each individual map using non-parametric random-permutation tests $(\mathrm{p}<0.05)$ (Figure 2B). Finally, we identified the most sensitive face regions at the group level using the 14 maps estimated at the subject level. Each voxel was tested using the non-parametric, one tailed, sign test (306405 tests, $p<0.05)$ while the Family Wise Error Rate (FWER) was controlled using the maxT/minP multiple testing procedure ${ }^{65}$. This process led to a statistical non-parametric mapping of the evoked activity in the superior temporal region (Figure 2B) for each group. For all permutation-based tests the permuted values were, at the subject-level, the $m_{t}$ values. For visualization purposes only, we obtained a smoothed representation of the results with a spatial resolution of $5 \times 5$. This was calculated by bicubic interpolation to map the variation in intensity of the evoked activity studied as a function of the region focused by the subjects (Figure 2B).

\section{Analysis 2: Evoked activity a priori}

First, we generated a "facial cue" map regressor from neurotypical group data provided by Lio et al. ${ }^{1}$. Then, for each participant, we applied a multiple linear regression model to assess how much the multichannel EEG activity was able to decode the "facial cue map" over time (Figure 1C).

\section{Facial cue map regressor}

We generated the "facial cue" map regressor by applying a sagittal symmetry and subsampling the cortical sensitivity map generated by neurotypical participants as in Lio et al.'s study ${ }^{1}$ (See Figure $3 \mathrm{~A}$ left). 
The obtained regressor implies that evoked activity is maximal in the eyes and eyebrows region and gradually decrease over the nose and mouth and other face regions to reach a minimum outside the face.

\section{Multiple linear regression of EEG activity}

With this analysis we avoided making any spatial modeling assumptions with regard to sources or anatomy and relied entirely on the statistics of the observed data and its covariation with observable stimuli. Here, we denote $\mathbf{x}(t)$ as the vector of multidimensional EEG data at time $t$. A linear projection combines the information from the multiple sensors into a single channel whose time course can be analyzed with conventional methods. We aimed to find a weighted matrix, w, which could discriminate, at the single-trial level, among the fixated areas over the face, the activity related to the face components. We calculated a multiple linear regression model at every time point to predict multichannel EEG activity based on the facial cue map regressor.

\section{Results}

\section{Behavioral results}

In the gender task, WS patients correctly identified only $67 \%$ of faces while neurotypical subjects and ASD were able to reach $91 \%$ and $89 \%$ of correct responses, respectively. Although WS patients were less accurate at identifying gender compared to the other groups (WS vs ASD: $t(27)=5.25, p<0.001$ and WS vs neurotypical: $t(27)=-5.61, p<0.001)$ they were able to perform above chance-level $(t(13)=4.17, p<0.001)$. Reduced identification performance in patients with WS may be explained by deficiencies in both general working memory and face processing capacities 66,67 , The accuracy did not vary as a function of the face part $(F(24,975)=0.7, p=0.85)$ and face parts did not interact with the group effect $(F(48,975)=0.74$, $p=0.91)$.

\section{EEG results}

\section{Analysis 1: Single trial evoked activity based on spatio-temporal filter}

Using a Group Blind Source Separation (gBSS) analysis and cluster-permutation test Lio et al. ${ }^{1}$, (see Method) revealed a significant source in neurotypical participants consisting in a large evoked activity with a maximum at $240 \mathrm{~ms}$ after the stimulus onset, and a source localization showing a maximum of activity around the lateral fissure (MNI coordinates $=\mathrm{X}:+65-65 \mathrm{Y}:-20 \mathrm{Z:}: 10)$ and a local maximum around the inferior temporal sulcus (MNI coordinates $=X:+60-60 \mathrm{Y}:-40 \mathrm{Z}:-20)$.

Based on the scalp topography of this source occurring between $200-300 \mathrm{~ms}$, we built a spatio-temporal filter and used it to extract evoked single-trial activity in patients with WS. For each participant, a map was generated indicating the level of evoked activity as a function of each of the 25 viewed face areas 
(See Figure 2 and method). This analysis allowed us to highlight how cortical sensitivity in the STS occurring at $200-300 \mathrm{~ms}$ is affected by different face regions. Finally, we performed group analysis to locate, in each group, which face region evoked the more pronounced activity.

Group results are shown in Figure 2. We report the degree of evoked activity in the STS measured as a function of the face region attended by participants with maximum and significant $(p<0.05$ FWER corrected) areas of evoked activity. As reported by Lio et al. ${ }^{1}$ in neurotypical subjects, the evoked activity is eye-sensitive. These subjects produced a maximal evoked activity in the upper part of the face $(p<0.05$ FWER corrected, indicated by blue tiles over the right small face), over the eyes and eyebrows, and a local minimum in the left and right lower corners, outside the face area. Patients with ASD showed an atypical pattern with significant activity on the nose region and cheek $(p<0.05$, FWER corrected, see Figure 2 , bottom left). This previously reported result is consistent with neurotypical eye tracking behavior in face perception tasks whereby more attention is paid to the eye region than to the mouth areas as well as with previous eye-tracking studies of how ASD patients attend to faces ${ }^{15,68}$.

WS patients showed an activation map with significant activity over the eyes, eyebrows and nose region $(p<0.05$, FWER corrected, see Figure 2, bottom center) and local minimum in the left and right lower corners and outside the face area. The pattern of evoked activity at $260 \mathrm{~ms}$ in the STS mirror that of patients with ASD and matches that of neurotypical participants.

\section{Analysis 2: Prediction of evoked activity with face cue map spatial regressor}

The maximal evoked activity for neurotypical participants is reminiscent of a T-shape over the face: the eyebrows, eyes, nose and mouth (and these face parts carry rich source of social information), whereas we observe lower activity for other areas of the face that are considered to have little social salience. Consistently, the minimum of the activity was recorded when patients' eyes were forced to look outside the face. We further built a face cue map spatial regressor generated by applying a sagittal symmetry and subsampling the cortical sensitivity map obtained by the neurotypical population and label this regressor a 'face cue map'.

Then, for each subject a temporal signal of the Fischer statistic denoting the quality of decoding obtained between $0-1000 \mathrm{~ms}$ was generated and compared across groups. We report each decoding signal (F-stat) scaled between zero ( $\mathrm{min}$ ) and one (max) for each subject and averaged for the group analysis in Figure 3.

At the group level, the F-stat value indicates that the decoding of the signal by the 'face cue map' was significant around the $150-350 \mathrm{~ms}$ time range after stimulus onset in all three groups $(p<0.05$, FWER corrected, see Figure 3 bottom left). The decoding curve in neurotypical yielded two marked peaks, one appearing at $170 \mathrm{~ms}$ after stimulus onset, and a second appearing at $260 \mathrm{~ms}$ after stimulus onset. This 
suggests the presence of two independent and/or interacting processes that are dependent on facial cues.

Although two response peaks with similar timing were identified in all groups, we observed different response level for these two processes for the two patients' groups (See Figure 3A). The ASD population showed a significantly greater decoding peak than the WS population at the earlier timing $(170 \mathrm{~ms}$ post stimulus onset, ASD > WS - $p<0.05$ FWER corrected). A reversed pattern can be found at the latter decoding peak: patients with WS showed a significantly greater decoding peak than the ASD population at the later timing ( $260 \mathrm{~ms}$ post-stimulus onset, WS>ASD $p<0.05$ FWER corrected).

For each group, we further tested which of the two peaks were maximal at the subject level. The temporal distribution of the maximum peak of decoding can be found in Figure 3B. These temporal distributions were found to differ between groups (Kruskall-Wallis, $\left.\operatorname{Chi}^{2}(2,39)=11.9, p=0.0026\right)$. For neurotypical participants, we found that the distribution of the maximum peak of decoding had a bimodal distribution with a dominance for the second process. This distribution indicates that face decoding goes preferentially through a social decoding in neurotypical participants. ASD patients also showed a bimodal distribution but the early process at $170 \mathrm{~ms}$ was significantly prominent. Consistent with behavioral results, this pattern suggests that faces' related processes within the ventral regions are wellpreserved. Finally, WS patients showed a strictly unimodal distribution such that activity was focused around the second process only.

Post-hoc analyses revealed a clear dissociation between both the WS and ASD patients ( $p<0.01$ FWER corrected) and the WS group and neurotypical population ( $p<0.05$ FWER corrected). This result combined with the low performance of WS patients in the gender identification task, suggests a preferential use of the dorsal face processing regions during face processing tasks compared to the ventral and is consistent with social cognition biases found in this syndrome.

\section{Prediction of EEG decoding with behavioral measures in neurotypical participants}

To assess whether the decoding timing was related to behavioral variables in neurotypical participants, we performed a multiple linear regression (stepwise) to predict the maximal onset of decoding based on accuracy in the task, visuospatial reasoning abilities (matrix), language reasoning abilities (similarities) and age in neurotypical participants. A significant regression equation was found $F(1,13)=8.56, p=0.013$, with an $\mathrm{R}^{2}$ of 0.42 . Visuospatial reasoning abilities (matrix score) were the only significant predictor of the maximal onset of decoding $(t=-2.9, p=0.013)$.

\section{Discussion}


The present study confirms the previous results reported by Lio et al. ${ }^{1}$ and extend these findings by dissociating the time course of neural processes involved in face perception in WS and ASD patients.

Our analyses confirm that activity in neurotypical participants can be evoked by viewing the eye region of a natural face stimulus. Furthermore, the evoked activity originates bilaterally in the superior temporal regions and peaks at $260 \mathrm{~ms}$ after stimulus onset. Notably, we show that the evoked STS eye sensitive response is also present in the WS group, contrary to what is observed in ASD participants by Lio et al'. This reveals a dissociation among these two patients' groups and suggest that although both syndromes are associated with social disturbances, their impairment at the neural level may have a different origin.

When examining face processing related neural signals along the occipito-temporal stream, we found two peaks in neurotypical participants: the first at $170 \mathrm{~ms}$, an early signal known to be implicated in low-level face features, the second at $260 \mathrm{~ms}$, a late component implicated in decoding salient face social cues ${ }^{1}$. Remarkably, both components were found distinctly impaired and preserved in WS and ASD. In WS, we could weakly decode the $170 \mathrm{~ms}$ signal probably due to their relatively poor ability to process faces' morphology while the late $260 \mathrm{~ms}$ component shown to be eye sensitive was highly significant. The reverse pattern was observed in ASD participants who showed neurotypical like early $170 \mathrm{~ms}$ evoked activity but impaired late evoked $260 \mathrm{~ms}$ signal.

Hence, we report a functional double dissociation between early (170 ms) and late (260ms) neurophysiological responses to faces in two neurodevelopmental disorders known to offer dissociable and opposing behaviors in facial processing and social cognition. Face processing is thought to involve the ventral pathway (through the OFA and FFA) for low-level features that are generally invariant, such as gender, age and identity, while the dorsal pathway (through the STS) is thought to process facial movements related to emotional expressions, gaze cues or intentions ${ }^{69-71}$. The latter is also considered to be the gateway to an extended system of social perception encompassing the orbitofrontal cortex and the amygdala ${ }^{38}$. Indeed, the present finding supports the hypothesis that early (170ms) visual processing of faces in the temporo-occipital area (FFA) and late social processing of faces in the temporal sulcus (i.e. STS) are functionally dissociable (Figure 4).

The present data have theoretical implications for understanding WS and their characteristic atypical processing of social cues, particularly faces. Indeed, our findings suggest that patients with WS, when viewing the eyes of a face, lack specificity in their early neurophysiological response. Early neurophysiological responses to faces at $170 \mathrm{~ms}$ are evoked by facial features ${ }^{22,23}$. This is thought to allow a consistent description of facial parts to be computed and processed in an holistic manner in the fusiform face area at $170 \mathrm{~ms} 24,25,28$.

Previous $\mathrm{fMRI}$ studies have shown that, during face perception, less activity can be observed in patients with WS than in neurotypical participants in areas specialized in early processing of face parts ${ }^{54}$. Our data are consistent with these results and suggest that the FFA-driven process of combining parts of the face into a holistic representation may be specifically impaired in WS.

Page $12 / 26$ 
We show that early responses in patients with WS are not modulated by the type of facial features on display (i.e., eyes, eyebrow, mouth compared to other face-parts). Such an absence of modulation may suggest that patients with WS make little distinction between facial features and their possible relevance as a means for facial identification and subsequent holistic processing. Indeed, WS patients show increased activity at N170 amplitudes when viewing trustworthy versus untrustworthy faces ${ }^{72,73}$.

In the present study, we applied blind source separation techniques to analyze high-resolution EEG data. EEG data were spatially processed to ensure that the source occurring at $170 \mathrm{~ms}$ did not originate in the STS. We also show that WS patients produce robust responses to late processing of facial cues at $260 \mathrm{~ms}$ in the STS. This observation is consistent with previous fMRI studies reporting greater STS activity in WS patients compared to patients with anxiety disorders while performing a facial perception task $^{54}$.

Unlike the study by Shore et al. ${ }^{72}$, the altered neural response we found cannot be attributed to different patterns of eye fixation when looking at the face stimulus ${ }^{16,55,74}$. Indeed, our method assessed the neurophysiological response to specific regions of the face presented to the foveae of each participant. This experimental modulation is extremely relevant to WS, as it has been hypothesized that the facial scanning behaviour of these patients is influenced by a general difficulty in disengaging attention from salient targets, known as "sticky fixation" 75,76 .

Our findings support two alternative interpretations. First, the role of the STS, which is typically implicated in late social processing of faces in neurotypical participants ${ }^{38}$, may be upregulated in patients with WS, thus leading to their characteristic highly social behavior. As a consequence, early analytical computations performed by the FFA ${ }^{20}$ would be bypassed, thus contributing to reduced performance in holistic face processing. Such interpretation would be coherent with reports of increased cortical thickness in the superior temporal gyrus of patients with WS ${ }^{53}$. Such interpretation would also fit well with the frontal dysfunction hypothesis, expressed as an inability to inhibit impulsive responses to social information ${ }^{44}$ and the amygdala dysfunction hypothesis, expressed by atypical processing of emotional information relevant to social interactions ${ }^{45-47}$. Children with WS are known to respond differently to social cues conveyed by the face ${ }^{8,77-79}$, and to process emotional cues atypically resulting in poor processing of social information from faces ${ }^{80}$. If the early analytical computation is by-passed it could explain why soft signs are not typically captured by patients with WS. Yet, in the present study, we do not evidence any atypical performance over the late component in patients with WS.

A second interpretation may be that early visual activity in temporo-occipital areas is dysfunctional in WS and are thus unable to discriminate specific facial features. This interpretation is supported by previous reports showing that the FFA is both enlarged ${ }^{49}$ and structurally altered ${ }^{45}$ in these patients. In our study, the poor decoding of the facial cue regressor may also be related to their poor visuospatial abilities. In fact, in neurotypical participants the onset of the maximal decoding of the facial cue map regressor was predicted by visuospatial reasoning abilities (in the matrix subtest). That is, participants who showed 
poor visuospatial abilities where more likely to exhibit a late peak than those with good performance. Therefore, it is possible that the first facial process in the ventral part of patients' brain poorly decode facial features due to poor visuospatial abilities. This could also explain why WS patients showed a lower performance in our gender task given their impairments in the perceptual holistic processes of faces. Subsequent cascading developmental consequences or compensatory strategies may induce a secondary upregulation of facial processing in other functional structures, namely the STS in this case, thus, leading to a concomitant increase in the social processing of faces. The role of cascading developmental consequences has already been theorized to explain atypical behaviors in other domains associated with Williams's syndrome (such as mathematical impairments explained by their poor visuospatial abilities, ${ }^{81}$ ). Such hypothesis is consistent with the atypical visual processing of faces from early infancy in WS ${ }^{72,82}$.

Overall, the notion of cascading pathways between early $(170 \mathrm{~ms})$ and late $(260 \mathrm{~ms})$ processes remains putative and could be coincidental. However, we argue that their development is closely intertwined. Still, whether the early neurophysiological face processing deficit in WS emerges from the neurodevelopmental consequence of the disorder or as the result of the poor social interaction exhibited by WS patients remains an unresolved question.

\section{What are the interventional implications of these results? Targeting STS and FFA in neurofeedback for WS and ASD}

Overall, our results raise the possibility of developing functional and behavioural rehabilitative procedures for both WS and ASD based on neurofeedback, a procedure in which self-regulation is stimulated by providing online feedback of neural activity to participants ${ }^{83,84}$. By controlling specific neural substrates, it can be possible to pinpoint and modify specific behaviours. Due to its high spatial resolution, $f M R I$ is usually used to target specific cerebral structures with neurofeedback ${ }^{85,86}$. Most procedures using EEG trigger training in EEG signal coherence or frequency ${ }^{87}$. Rather than being based on modulation of disorder-specific biomarkers, most current EEG neurofeedback protocols are based on the modulation of a few spontaneous brain rhythms, mainly defined by the frequency of their oscillation ${ }^{83,84}$. This strategy is widespread because spontaneous brain rhythms demonstrate a high signal-to-noise ratio in EEG recordings and can also be disrupted in some mental disorders (e.g., ADHD ${ }^{88}$ ). However, within the broad field of psychiatric disorders, such methods lack specificity.

Here, ERPs could be used to feeding back levels of local cortical arousal back to patients with the purpose of improving the self-regulation of cortical excitability in the specific structure related to the disorder ${ }^{89}$. In order to respond to the specific needs of patients, training would be provided beforehand. Indeed, it has been demonstrated that patients with severe intellectual impairments, like some patients with ASD and WS, are able to follow training procedures to enhance the effectiveness of neurofeedback 90 . 
Our results provide unique and specific biomarkers associated with face processing deficits in ASD and WS. In light of these findings, it may be beneficial to explore whether neurofeedback can help children with ASD or WS to enhance STS or FFA activity during face processing. Here, the goal would simply be to manipulate neural activity in the STS or FFA in order to rebalance social processing of face related to this structure, i.e., the identification of facial features. Neural activity in the STS or FFA could be measured by high-density EEG which could then be represented in real time, through visual, audio or other means, back to participants while the focused area on the face different facial areas (either social, eyes, nose, brows, mouths or not) to facilitate self-regulation of cortical excitability underlying the specific behaviour. This might be achieved with the use of one signal for the FFA (e.g., visual) and another signal for the STS (e.g., auditory) to assist patients in reassigning their attention to facial features for the purposes of identity recognition or social cognition ${ }^{17}$.

One would expect such training to facilitate the identification of a neural strategy that can be employed by patients when viewing faces. Indeed, the idea would be to train patients both at the behavioural level to pay attention to socially relevant facial cues but to also to train them to engage their FFA and STS when viewing specific facial features (e.g., eye, eyebrows, mouth). The long-term goal could be that both groups of patients would engage the ventral and-dorsal areas in a more balanced way during social interactions.

More generally, facial and social skills are essential abilities for navigating in our modern society. With the ongoing pandemic, the ability to decode social information from faces on a screen or from the eye region (as faces are partially occluded with masks) has recently become one of the main tools in social interactions ${ }^{91}$. This ability relies on a complex cerebral network ${ }^{28}$. Double dissociations using fine spatiotemporal analysis of this network provide essential evidence for understanding what, where and when neurocomputations are performed in our brain. In patients with Williams syndrome, poor decoding of facial features by low-level visual processing can result in some of the observed social symptoms that can have catastrophic influences on their lives. Future works may examine whether, in turn subtle changes in the balance of this network, through self-regulated neurofeedback training, can attenuate their characteristic social behaviour and related symptoms of social dysfunctions, such as anxiety.

\section{Abbreviations}

ADHD: Attention Deficit Hyperactivity Disorder; ASD: Autism Spectrum Disorder; ERP: Event-related Potentials; FFA: Fusiform Face Area; fMRI: functional Magnetic Resonance Imaging; gBSS: group Brain Source Separation; FWHM: Full Width at Half Maximum; FWER: Family-Wise error Rate; IQ: Intellligence quotient; NEPSY: A Developmental NEuroPSYchological Assessment; OFA: Occipital Face Area; STS: Superior Temporal Sulcus; pSTS: Posterior Superior Temporal Sulcus; ROI: Region of Interest; WS: Williams Syndrome; WAIS : Weschler Adult Intelligence Scale; WISC-V: Weschler Intelligence Scale for children -V.

\section{Declarations}




\section{Acknowledgment and Funding}

This research was supported by CNRS, Vinatier Hospital, a Young researcher Grant from Lyon University to AG and by Labex Cortex (ANR-11-LABEX-0042) grant from the University of Lyon I within the program "Investissement d'Avenir" to AS. The manuscript is accessible on Biorxiv:

https://www.biorxiv.org/content/10.1101/2021.04.07.438774v1.

\section{Conflict of interest}

The authors report no competing interests.

\section{Data availability}

The conditions of our ethics approval do not permit public archiving of patients' data. Public access to data can be obtained after permission of the French ethical committee (CPP Sud-Est IV) who granted ethical approval to this project (NPP:16/018, N ID RCB: 2014-A01894-43, Promoted by CNRS).

\section{Author contributions}

A. Gomez, G. Lio, M. Costa, A. Sirigu and C. Demily designed the study. C. Demily performed genetic and patients' clinical assessment. A. Gomez and M. Costa performed testing and experimental data collection. G. Lio designed EEG analysis. A. Gomez, M. Costa and G. Lio performed data analysis. A. Gomez wrote the manuscript and A. Sirigu and C. Demily provided critical revisions. All authors approved the final version of the manuscript for submission.

\section{References}

1. Lio G, Corazzol M, Fadda R, Doneddu G, Demily C, Sirigu A. A neural marker of eye contact highly impaired in autism spectrum disorder. bioRxiv. Published online January 1 , 2021:2021.03.29.433074. doi:10.1101/2021.03.29.433074

2. American Psychiatric Association, Association AP. Diagnostic and Statistical Manual of Mental Disorders (DSM-5®). American P.; 2013.

3. Jones W, Klin A. Attention to eyes is present but in decline in 2-6-month-old infants later diagnosed with autism. Nature. Published online 2013. Accessed January 30, 2014. http://www.nature.com/nature/journal/vaop/ncurrent/full/nature12715.html? WT.mc_id=TWT_NatureNeuro

4. Jones W, Carr K, Klin A. Absence of preferential looking to the eyes of approaching adults predicts level of social disability in 2-year-old toddlers with autism spectrum disorder. Arch Gen Psychiatry. 2008;65(8):946-954. doi:10.1001/archpsyc.65.8.946 
5. Korenberg JR, Xiao-Ning C, Hirota H, et al. Genome structure and cognitive map of Williams syndrome. J Cogn Neurosci. 2000;12(Supplement, Number 1):89-107.

6. Bellugi U, Jarvinen-Pasley A, Doyle TF, Reilly J, Reiss AL, Korenberg JR. Affect, social behavior, and the brain in Williams syndrome. Curr Dir Psychol Sci. 2007;16(2):99-104. http://www.lcn.salk.edu/publications/2007/bellugi_pasley_curr_dir_2007.pdf

7. Zitzer-Comfort C, Doyle T, Masataka N, Korenberg J, Bellugi U. Nature and nurture: Williams syndrome across cultures. Dev Sci. 2007;10(6):755-762. doi:10.1111/j.1467-7687.2007.00626.x

8. Doyle TF, Bellugi U, Korenberg JR, Graham J. "Everybody in the world is my friend" hypersociability in young children with Williams syndrome. Am J Med Genet A. 2004;124A(3):263-273. doi:10.1002/ajmg.a.20416

9. Pavlova MA, Heiz J, Sokolov AN, Barisnikov K. Social Cognition in Williams Syndrome: Face Tuning. 2016;7(August):1-8. doi:10.3389/fpsyg.2016.01131

10. Jarvinen A, Ng R, Bellugi U. Autonomic response to approachability characteristics, approach behavior, and social functioning in Williams syndrome. Neuropsychologia. 2015;nov(78):159-170. doi:10.1002/cncr.27633.Percutaneous

11. Mervis CB, Morris CA, Klein-Tasman BP, et al. Attentional Characteristics of Infants and Toddlers With Williams Syndrome During Triadic Interactions. Dev Neuropsychol. 2003;23(1-2):243-268. doi:10.1080/87565641.2003.9651894

12. Bellugi U, Lichtenberger L, Jones W, Lai Z, St. George M. I. The Neurocognitive Profile of Williams Syndrome: A Complex Pattern of Strengths and Weaknesses. J Cogn Neurosci. 2000;12(supplement 1):7-29. doi:10.1162/089892900561959

13. Paul BM, Stiles J, Passarotti A, Bavar N, Bellugi U. Face and place processing in Williams syndrome: Evidence for a dorsal-ventral dissociation. Neuroreport. 2002;13(9):1115-1119. doi:10.1097/00001756-200207020-00009

14. Tager-Flusberg H, Plesa-Skwerer D, Faja S, Joseph RM. People with Williams syndrome process faces holistically. Cognition. 2003;89(1):11-24. doi:10.1016/S0010-0277(03)00049-0

15. Riby DM, Doherty-Sneddon G, Bruce V. The eyes or the mouth? Feature salience and unfamiliar face processing in Williams syndrome and autism. Q J Exp Psychol. 2009;62(1):189-203. doi:10.1080/17470210701855629

16. Hirai M, Muramatsu Y, Mizuno S, Kurahashi N, Kurahashi H, Nakamura M. Typical visual search performance and atypical gaze behaviors in response to faces in Williams syndrome. J Neurodev Disord. 2016;8(1):1-14. doi:10.1186/s11689-016-9172-7

17. Lee K, Anzures G, Quinn PC, Pascalis O, Slater AM. Development of Face Processing Expertise. Oxford Handb Face Percept. 2012;(8):1-15. doi:10.1093/oxfordhb/9780199559053.013.0039

18. Lee K, Eskritt M, Symons LA, Muir D. Children's use of triadic eye gaze information for "mind reading". Dev Psychol. 1998;34(3):525-539. doi:10.1037/0012-1649.34.3.525

19. Kanwisher N, McDermott J, Chun MM. The fusiform face area: a module in human extrastriate cortex specialized for face perception. J Neurosci. 1997;17(11):4302-4311. 
http://www.ncbi.nlm.nih.gov/pubmed/9151747

20. Kanwisher N, Tong F, Nakayama K. The effect of face inversion on the human fusiform face area. Cognition. 1998;68(1):1-11. doi:10.1016/S0010-0277(98)00035-3

21. Haxby J, Horwitz B, Ungerleider LG, Maisog JM, Pietro P, Grady CL. The Functional Organization of Human Extrastriate Cortex: A PET-rCBF Study of Selective Attention to Faces and Locations. $J$ Neurosci. 1994;14(11):6336-6353.

22. Allison T. Electrophysiological Studies of Human Face Perception. I: Potentials Generated in Occipitotemporal Cortex by Face and Non-face Stimuli. Cereb Cortex. 1999;9(5):415-430. doi:10.1093/cercor/9.5.415

23. Bentin, S., Allison, T., Puce, A., Perez, E., \& McCarthy, G. (1996). Electrophysiological studies of face perception in humans. Journal of cognitive neuroscience, 8(6), 551-565.

24. Gauthier I, Tarr MJ, Moylan J, Skudlarski P, Gore JC, Anderson AW. The fusiform face area is part of a network that processes faces at the individual level. J Cogn Neurosci. 2000;12(3):495-504.

25. Pitcher D, Walsh V, Duchaine B. The role of the occipital face area in the cortical face perception network. Exp Brain Res. 2011;209(4):481-493. doi:10.1007/s00221-011-2579-1

26. Sadeh B, Podlipsky I, Zhdanov A, Yovel G. Event-related potential and functional MRI measures of face-selectivity are highly correlated: A simultaneous ERP-fMRI investigation. Hum Brain Mapp. 2010;31(10):1490-1501. doi:10.1002/hbm.20952

27. Mattavelli G, Pisoni A, Romero Lauro LJ, et al. TMS-EEG approach unveils brain mechanisms underlying conscious and unconscious face perception. Brain Stimul. 2019;12(4):1010-1019. doi:10.1016/j.brs.2019.02.022

28. Yovel G. Neural and cognitive face-selective markers: An integrative review. Neuropsychologia. 2016;83:5-13. doi:10.1016/j.neuropsychologia.2015.09.026

29. Winston JS, Henson RNA, Fine-Goulden MR, Dolan RJ. fMRI-adaptation reveals dissociable neural representations of identity and expression in face perception. $J$ Neurophysiol. 2004;92(3):1830-1839. doi:10.1152/jn.00155.2004

30. Pitcher D, Dilks DD, Saxe RR, Triantafyllou C, Kanwisher N. Differential selectivity for dynamic versus static information in face-selective cortical regions. Neuroimage. 2011;56(4):2356-2363. doi:10.1016/j.neuroimage.2011.03.067

31. Puce A, Allison T, Bentin S, Gore JC, McCarthy G. Temporal cortex activation in humans viewing eye and mouth movements. J Neurosci. 1998;18(6):2188-2199. doi:10.1523/jneurosci.18-06-02188.1998

32. Schobert AK, Corradi-Dell'Acqua C, Frühholz S, van der Zwaag W, Vuilleumier P. Functional organization of face processing in the human superior temporal sulcus: A 7T high-resolution fMRI study. Soc Cogn Affect Neurosci. 2018;13(1):102-113. doi:10.1093/scan/nsx119

33. Burra N, Baker S, George N. Processing of gaze direction within the N170/M170 time window: A combined EEG/MEG study. Neuropsychologia. 2017;100(April):207-219.

doi:10.1016/j.neuropsychologia.2017.04.028

Page 18/26 
34. Slotnick. Source localization of ERP generators. In: Handy T, ed. Event-Related Potentials: A Methods Handbook. MIT Press. ; 2004:149-166.

35. Nguyen VT, Cunnington R. The superior temporal sulcus and the N170 during face processing: Single trial analysis of concurrent EEG-fMRI. Neuroimage. 2014;86:492-502. doi:10.1016/j.neuroimage.2013.10.047

36. Horovitz SG, Rossion B, Skudlarski P, Gore JC. Parametric design and correlational analyses help integrating $\mathrm{fMRI}$ and electrophysiological data during face processing. Neuroimage. 2004;22(4):1587-1595. doi:10.1016/j.neuroimage.2004.04.018

37. Henson R, Goshen-Gottstein Y, ... TG-C, 2003 undefined. Electrophysiological and haemodynamic correlates of face perception, recognition and priming. academic.oup.com. Accessed March 19, 2021. https://academic.oup.com/cercor/article-abstract/13/7/793/420416

38. Allison T, Puce A, McCarthy G. Social perception from visual cues: role of the STS regions. Trends Cogn Sci. 2000;4(7). doi:10.1016/S1364-6613(00)01501-1

39. Zilbovicius M, Meresse I, Chabane N, Brunelle F, Samson Y, Boddaert N. Autism, the superior temporal sulcus and social perception. Trends Neurosci. 2006;29(7):359-366. doi:10.1016/j.tins.2006.06.004

40. Dakin S, Frith U. Vagaries of visual perception in autism. Neuron. 2005;48(3):497-507. doi:10.1016/j.neuron.2005.10.018

41. Zilbovicius M, Boddaert N, Belin P, et al. Temporal lobe dysfunction in childhood autism: A PET study. Am J Psychiatry. 2000;157(12):1988-1993. doi:10.1176/appi.ajp.157.12.1988

42. Boddaert N, Chabane N, Gervais $\mathrm{H}$, et al. Superior temporal sulcus anatomical abnormalities in childhood autism: A voxel-based morphometry MRI study. Neuroimage. 2004;23(1):364-369. doi:10.1016/j.neuroimage.2004.06.016

43. Hotier S, Leroy F, Boisgontier J, et al. Social cognition in autism is associated with the neurodevelopment of the posterior superior temporal sulcus. Acta Psychiatr Scand. 2017;136(5):517525. doi:10.1111/acps.12814

44. Porter MA, Coltheart M, Langdon R. The neuropsychological basis of hypersociability in Williams and Down syndrome. Neuropsychologia. 2007;45(12):2839-2849. doi:10.1016/j.neuropsychologia.2007.05.006

45. Reiss AL, Eckert MA, Rose FE, et al. An Experiment of Nature: Brain Anatomy Parallels Cognition and Behavior in Williams Syndrome. 2004;24(21):5009-5015. doi:10.1523/JNEUROSCI.5272-03.2004

46. Meyer-Lindenberg A, Hariri A. Neural correlates of genetically abnormal social cognition in Williams syndrome. Nat .... Published online 2005. Accessed April 1, 2015. http://www.nature.com/neuro/journal/v8/n8/abs/nn1494.html

47. Bellugi U, Adolph R, Cassady C, Chiles M. Towards the neural basis for hypersociability in a genetic syndrome. Neuroreport. 1999;10(8):1653-1657.

48. Jones W, Bellugi U, Lai Z, et al. II. Hypersociability in Williams Syndrome. J Cogn Neurosci. 2000;12(supplement 1):30-46. doi:10.1162/089892900561968 
49. Golarai G, Hong S, Haas BW, et al. The fusiform face area is enlarged in Williams syndrome. J Neurosci. 2010;30(19):6700-6712. doi:10.1523/JNEUROSCI.4268-09.2010

50. Chiang MC, Reiss $A L$, Lee $A D$, et al. 3D pattern of brain abnormalities in Williams syndrome visualized using tensor-based morphometry. Neuroimage. 2007;36(4):1096-1109. doi:10.1016/j.neuroimage.2007.04.024

51. Sampaio A, Sousa N, Férnandez M, Vasconcelos C, Shenton ME, Gonçalves ÓF. MRI assessment of superior temporal gyrus in williams syndrome. Cogn Behav Neurol. 2008;21(3):150-156. doi:10.1097/WNN.0b013e31817720e4

52. Fung LK, Quintin EM, Haas BW, Reiss AL. Conceptualizing neurodevelopmental disorders through a mechanistic understanding of fragile $X$ syndrome and Williams syndrome. Curr Opin Neurol. 2012;25(2):112-124. doi:10.1097/WC0.0b013e328351823c

53. Green T, Fierro KC, Raman MM, Saggar M, Sheau KE, Reiss AL. Surface-based morphometry reveals distinct cortical thickness and surface area profiles in Williams syndrome. Am J Med Genet Part B Neuropsychiatr Genet. 2016;171(3):402-413. doi:10.1002/ajmg.b.32422

54. Binelli C, Muñiz A, Subira S, et al. Facial emotion processing in patients with social anxiety disorder and Williams-Beuren syndrome: An fMRI study. J Psychiatry Neurosci. 2016;41(3):182-191. doi:10.1503/jpn.140384

55. Mobbs D, Garrett a S, Menon V, Rose F, Bellugi U, Reiss a L. Anomalous brain activation during face and gaze processing in Williams syndrome. Neurology. 2004;62(11):2070-2076. doi:10.1212/01.WNL.0000129536.95274.DC

56. Mills DL, Alvarez TD, St George M, Appelbaum LG, Bellugi U, Neville H. III. Electrophysiological studies of face processing in Williams syndrome. J Cogn Neurosci. 2000;12 Suppl 1:47-64. doi:10.1162/089892900561977

57. Shore DM, Ng R, Bellugi U, Mills DL. Abnormalities in early visual processes are linked to hypersociability and atypical evaluation of facial trustworthiness: An ERP study with Williams syndrome. Cogn Affect Behav Neurosci. Published online 2017. doi:10.3758/s13415-017-0528-6

58. Weschler D. WISC-IV, Echelle d'intelligence de Weschler pour enfants et adolescents, Quatrième édition. Published online 2005.

59. Korkman M, Kirk U, Kemp SL. Bilan neuropsychologique de l'enfant (NEPSY). Published online 2003.

60. Oostenveld R, Praamstra P. The five percent electrode system for high-resolution EEG and ERP measurements. Clin Neurophysiol. 2001;112(4):713-719. doi:10.1016/S1388-2457(00)00527-7

61. Van Veen BD, Van Drongelen W, Yuchtman M, Suzuki A. Localization of brain electrical activity via linearly constrained minimum variance spatial filtering. IEEE Trans Biomed Eng. 1997;44(9):867-880. doi:10.1109/10.623056

62. Vrba J, Robinson SE. Signal processing in magnetoencephalography. Methods. 2001;25(2):249-271. doi:10.1006/meth.2001.1238

63. Albares M, Lio G, Criaud M, Anton J-L, Desmurget M, Boulinguez P. The dorsal medial frontal cortex mediates automatic motor inhibition in uncertain contexts: evidence from combined fMRI and EEG 
studies. Hum Brain Mapp. 2014;35(11):5517-5531. doi:10.1002/hbm.22567

64. Caldara R, Miellet S. iMap: a novel method for statistical fixation mapping of eye movement data. Behav Res Methods. 2011;43(3):864-878. doi:10.3758/s13428-011-0092-x

65. Westfall PH, Young SS, Wright SP. On Adjusting P-Values for Multiplicity. Biometrics. 1993;49(3):941. doi:10.2307/2532216

66. Rhodes SM, Riby DM, Park J, Fraser E, Campbell LE. Executive neuropsychological functioning in individuals with Williams syndrome. Neuropsychologia. 2010;48(5):1216-1226.

doi:10.1016/j.neuropsychologia.2009.12.021

67. Herwegen J Van. Williams syndrome and its cognitive profile: The importance of eye movements. Psychol Res Behav Manag. 2015;8:143-151. http://ovidsp.ovid.com/ovidweb.cgi?

$\mathrm{T}=\mathrm{JS} \& \mathrm{PAGE}=$ reference\&D$=$ ssyc11 \&NEWS $=\mathrm{N} \& A N=2015-26066-001$

68. Hernandez N, Metzger A, Magné R, et al. Exploration of core features of a human face by healthy and autistic adults analyzed by visual scanning. Neuropsychologia. 2009;47(4):1004-1012. doi:10.1016/j.neuropsychologia.2008.10.023

69. Haxby J V., Hoffman EA, Gobbini MI. The distributed human neural system for face perception. Trends Cogn Sci. 2000;4(6):223-233. doi:10.1016/S1364-6613(00)01482-0

70. Pascalis 0 , de Martin de Viviés X, Anzures G, et al. Development of face processing. Wiley Interdiscip Rev Cogn Sci. 2011;2(6):666-675. doi:10.1002/wcs.146

71. Bernstein M, Yovel G. Two neural pathways of face processing: A critical evaluation of current models. Neurosci Biobehav Rev. 2015;55:536-546. doi:10.1016/j.neubiorev.2015.06.010

72. Shore DM, Ng R, Bellugi U, Mills DL. Abnormalities in early visual processes are linked to hypersociability and atypical evaluation of facial trustworthiness: An ERP study with Williams syndrome. Cogn Affect Behav Neurosci. 2017;17(5):1002-1017. doi:10.3758/s13415-017-0528-6

73. Mills DL, Alvarez TD, St. George M, Appelbaum LG, Bellugi U, Neville H. III. Electrophysiological Studies of Face Processing in Williams Syndrome. J Cogn Neurosci. 2002;12(supplement 1):47-64. doi:10.1162/089892900561977

74. Hirai M, Muramatsu Y, Mizuno S, Kurahashi N, Kurahashi H, Nakamura M. Preserved search asymmetry in the detection of fearful faces among neutral faces in individuals with Williams syndrome revealed by measurement of both manual responses and eye tracking. $J$ Neurodev Disord. 2017;9(1):1-15. doi:10.1186/s11689-017-9190-0

75. D'Souza D, D'Souza H, Johnson MH, Karmiloff-Smith A. Concurrent relations between face scanning and language: A cross-syndrome infant study. PLoS One. 2015;10(10):1-26. doi:10.1371/journal.pone.0139319

76. Van Herwegen J. Williams syndrome and its cognitive profile: The importance of eye movements. Psychol Res Behav Manag. 2015;8:143-151. doi:10.2147/PRBM.S63474

77. Karmiloff-Smith A, D'Souza D, Dekker TM, et al. Genetic and environmental vulnerabilities in children with neurodevelopmental disorders. Proc Natl Acad Sci U S A. 2012;109 Suppl:17261-17265. doi:10.1073/pnas.1121087109 
78. Riby DM, Hancock PJB. Do faces capture the attention of individuals with Williams syndrome or Autism? Evidence from tracking eye movements. J Autism Dev Disord. 2009;39(3):421-431. doi:10.1007/s10803-008-0641-z

79. Karmiloff-Smith A, Klima E, Bellugi U, Grant J, Baron-Cohen S. Is there a social module? Language, face processing, and theory of mind in individuals with williams syndrome. $J$ Cogn Neurosci. 1995;7(2):196-208. doi:10.1162/jocn.1995.7.2.196

80. Gomez A, Costa M, Lio G, Demily C, Sirigu A. Face first impression of trustworthiness in Williams Syndrome: dissociating automatic vs decision based perception. Cortex. Published online 2020.

81. Eckert MA, Galaburda AM, Mills DL, Bellugi U, Korenberg JR, Reiss AL. The neurobiology of Williams syndrome: Cascading influences of visual system impairment? 2006;63:1867-1875. doi:10.1007/s00018-005-5553-x

82. D'Souza D, Cole V, Farran EK, et al. Face processing in Williams syndrome is already atypical in infancy. Front Psychol. 2015;6(JUN):1-9. doi:10.3389/fpsyg.2015.00760

83. Batail JM, Bioulac S, Cabestaing F, et al. EEG neurofeedback research: A fertile ground for psychiatry? Encephale. 2019;45(3):245-255. doi:10.1016/j.encep.2019.02.001

84. Sitaram R, Ros T, Stoeckel L, et al. Closed-loop brain training: The science of neurofeedback. Nat Rev Neurosci. 2017;18(2):86-100. doi:10.1038/nrn.2016.164

85. Linhartová $P$, Látalová A, Kóša $B$, Kašpárek T, Schmahl C, Paret C. fMRI neurofeedback in emotion regulation: A literature review. Neuroimage. 2019;193(June 2018):75-92.

doi:10.1016/j.neuroimage.2019.03.011

86. Okano K, Bauer CCC, Ghosh SS, et al. Real-time fMRI feedback impacts brain activation, results in auditory hallucinations reduction: Part 1: Superior temporal gyrus -Preliminary evidence-. Psychiatry Res. 2020;286(February). doi:10.1016/j.psychres.2020.112862

87. Omejc N, Rojc B, Battaglini PP, Marusic U. Review of the therapeutic neurofeedback method using electroencephalography: EEG neurofeedback. Bosn J Basic Med Sci. 2019;19(3):213-220. doi:10.17305/bjbms.2018.3785

88. Lambez B, Harwood-Gross A, Golumbic EZ, Rassovsky Y. Non-pharmacological interventions for cognitive difficulties in ADHD: A systematic review and meta-analysis. $J$ Psychiatr Res. 2020;120(September 2019):40-55. doi:10.1016/j.jpsychires.2019.10.007

89. Birbaumer N. Slow cortical potentials: Plasticity, operant control, and behavioral effects. Neuroscientist. 1999;5(2):74-78. doi:10.1177/107385849900500211

90. LaMarca K, Gevirtz R, Lincoln AJ, Pineda JA. Facilitating Neurofeedback in Children with Autism and Intellectual Impairments Using TAGteach. J Autism Dev Disord. 2018;48(6):2090-2100. doi:10.1007/s10803-018-3466-4

91. Freud E, Stajduhar A, Rosenbaum RS, Avidan G, Ganel T. The COVID-19 pandemic masks the way people perceive faces. Sci Rep. 2020;10(1):1-8. doi:10.1038/s41598-020-78986-9

\section{Tables}


Table 1: General cognitive abilities assessed in neurotypical participants and patients with WS.

\begin{tabular}{lccc}
$\begin{array}{l}\text { General cognitive } \\
\text { abilities tests }\end{array}$ & $\begin{array}{c}\text { Neurotypical } \\
(\mathbf{N}=14)\end{array}$ & $\begin{array}{c}\text { Patients with } \\
\text { WS (N=14) }\end{array}$ & $\begin{array}{c}\text { Two } \\
\text { samples t- } \\
\text { test } \text {-value }\end{array}$ \\
\hline Matrices & Mean (SD) & Mean (SD) & \\
Similarities & $11.9(2.8)$ & $3.8(2.9)$ & $<0.001$ \\
& $15.3(2.7)$ & $4.5(3.4)$ & $<0.001$ \\
Arrows & $12(2.5)$ & $2.1(2.1)$ & $<0.001$ \\
Auditory attention & $10.5(4.0)$ & $6.5(5.2)$ & 0.035 \\
\hline
\end{tabular}

\section{Figures}

A -Task (Lio et al. 2021).

High density

EEG
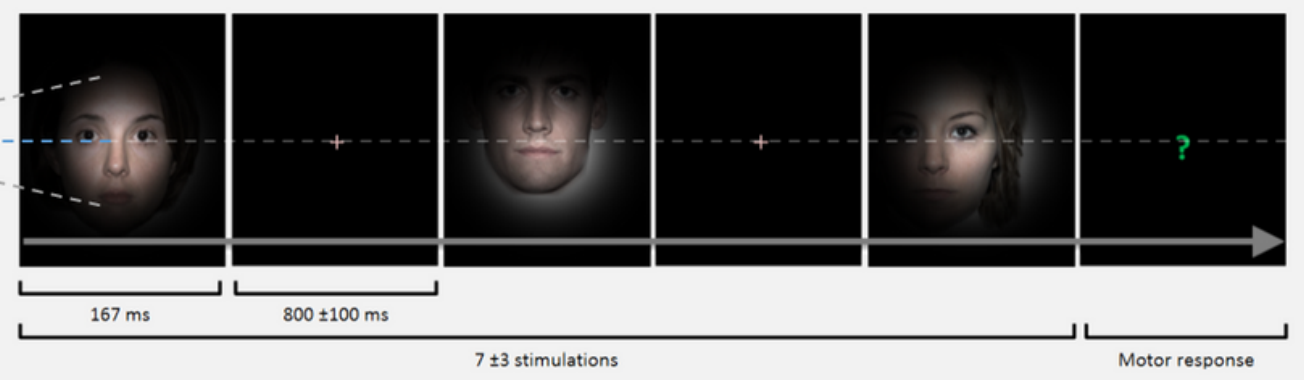

\section{B - Analysis 1}

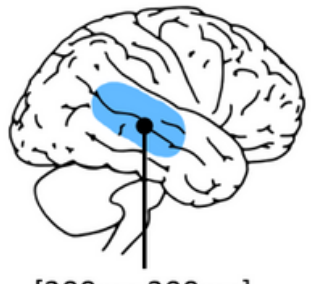

[200ms 300ms]

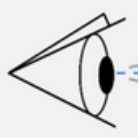

¿

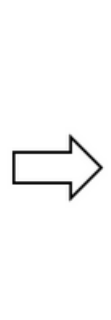

Spatio-temporal apriori

(Lio et al. 2021)

\section{C-Analysis 2}

$\checkmark$

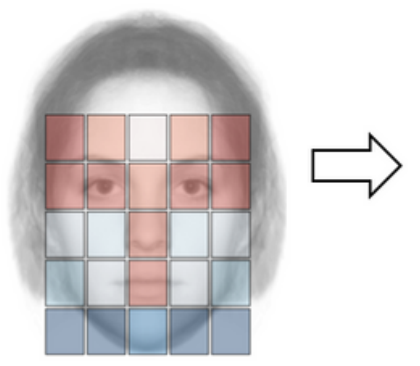

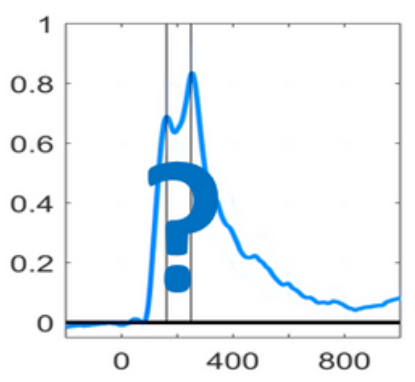

Evoked activity apriori

(Lio et al. 2021)

\section{Figure 1}

Methodological procedure summary. (A) Facial-cue task Participants were instructed to focus on a fixation cross and were then presented with a face stimulus masked by a Gaussian apodization window centered around the fixation cross $\left(\mathrm{FWHM}=10^{\circ}\right)$. The focused face area at the center of the screen was randomly drawn from a uniform distribution among 25 predetermined locations. A question mark was presented at random points (every $7 \pm 3$ trials) at which point participants were required to determine the gender of the last face stimulus that was viewed (left/right button press). Panel labels should be in bold 
font in the legend. (B) Analysis 1. The first analysis used a strong spatio-temporal a priori to build a map of cortical reactivity according to the face area that was focused on by each participant. Cortical reactivity was evoked in the region of the STS occurring 200-300ms after stimulus onset, as in Lio et al. The purpose of this analysis was to determine whether the neurotypical response to eye contact in this source was preserved in WS. (C) Analysis 2 . The second analysis aimed to identify changes in cortical reactivity associated with a "facial cue" regressor over time (built from the Lio et al. Exp .2 study, see method), and to identify when in time EEG activity can decode the "facial cue" map. This spatial regressor predicts a maximum evoked activity when participants focus on facial cues, with a progressive decay in response to other parts of the face and a minimum activity outside the face, as in the neurotypical population (See Figure 3A for details).

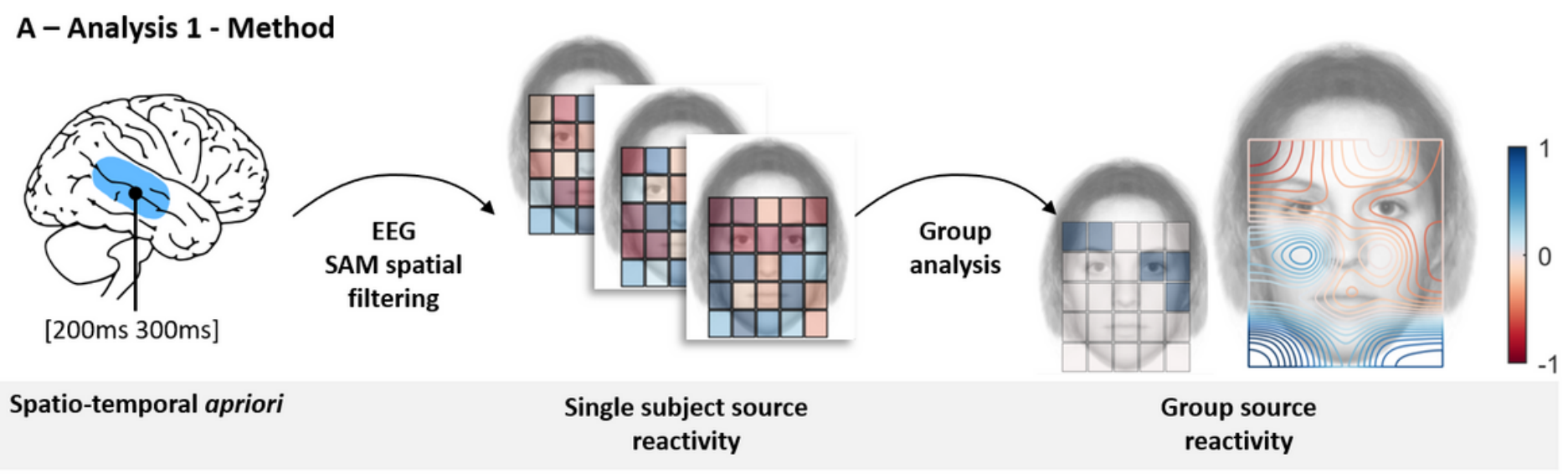

\section{B - Analysis 1 - Results}

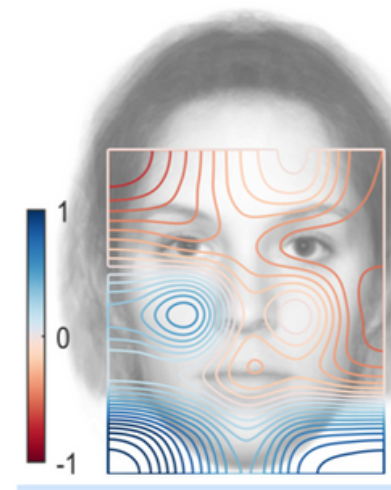

CTRL

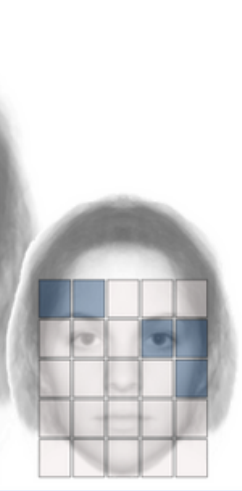

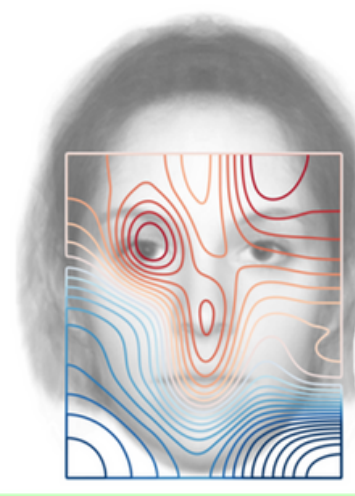

WS

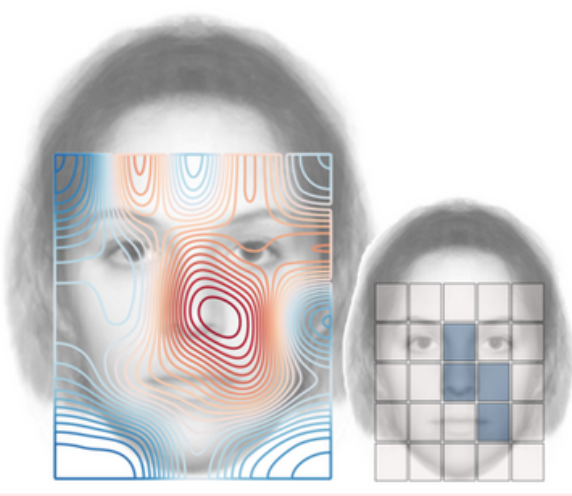

ASD

\section{Figure 2}

Method and results of the single trial evoked activity analysis. (A) Signal processing pipeline of this analysis 1. First, a spatial-filter was built to extract single-trial activity evoked between $200 \mathrm{~ms}$ and $300 \mathrm{~ms}$ in the superior temporal region at the single subject and group-level. (B) Results of evoked activity mapped over a face area for the neurotypical participants (CTRL, in blue, on the left), patients with Williams-Beuren syndrome (WS, in green, in the middle); and patients with autistic spectrum disorders (ASD, in red, on the right). For each group, the large face on the left (with a Red-Blue color scheme) shows 
a contour plot of interpolated results of the degree of evoked activity in the STS measured as a function of the face region attended to by participants. The small face on the right (with blue tiles) shows tiles which represent maximum and significant ( $p<0.05$ FWER corrected) areas of evoked activity. Activity in young neurotypicals confirms that activity was eye-sensitive, as found previously in adults (Lio et al. Exp. 2). This was maximal in the upper part of the face, over the eyes and eyebrows, and decreased to reach a local minimum in the left and right lower corners, outside the face area. WS patients showed a similar activation map with significant activity over the eyes, eyebrows and nose region. ASD showed an atypical pattern with significant activity on the nose region and cheek.

\section{A - Analysis 2 - Method}

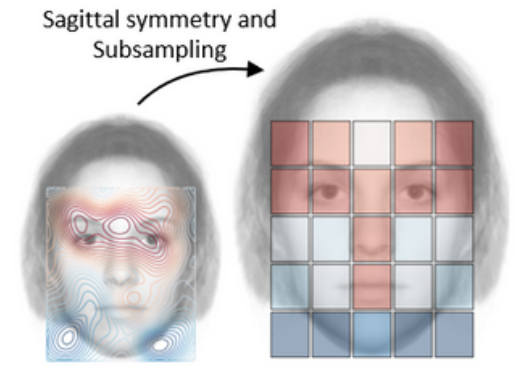

Evoked activity apriori

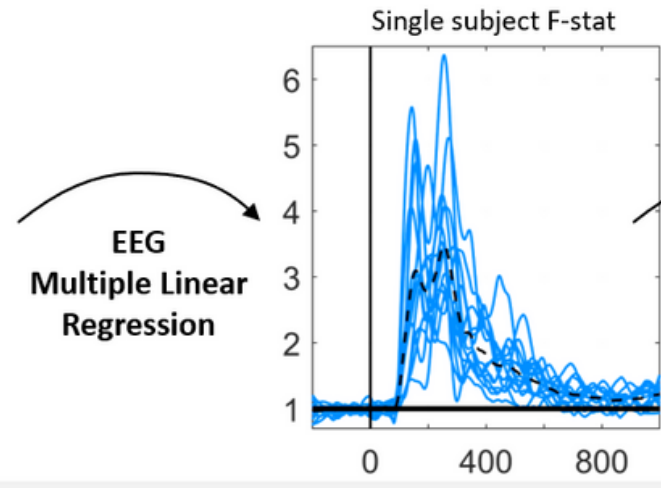

Single subject decoding
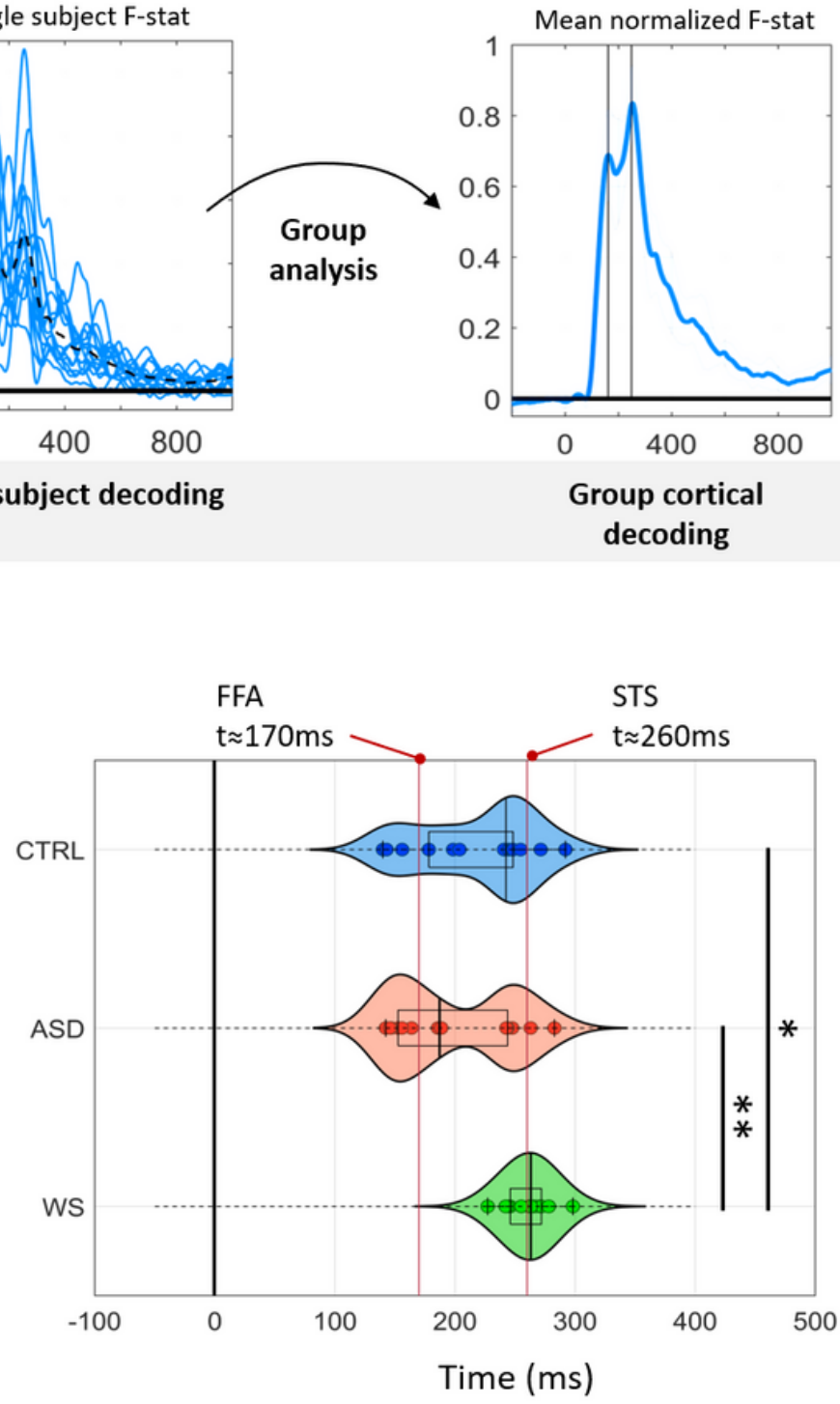

Figure 3

Prediction of evoked activity with face cue map spatial regressor. (A) Signal processing pipeline for the second analysis. The 'socially relevant face cue map' regressor was generated from the neurotypical evoked activity (applying a sagittal symmetry and subsampling) (left part). Then, a multiple linear regression is applied and the Fischer statistic denoting the quality of decoding obtained at each time 
point between $0-1000 \mathrm{~ms}$ is generated for each subject (see dotted lines, on the middle graph). Finally, each decoding signal (F-stat) scaled between zero ( $\mathrm{min}$ ) and one ( $\max$ ) for each subject was averaged for the group analysis (right graph). (B) Results from decoding the facial-cue map for the neurotypical participants (CTRL, in blue), patients with Williams-Beuren syndrome (WS, in green) and patients with autistic spectrum disorders (ASD, in red). On the left graph, F score for the decoding of each group as a function of time. Significant $F$ values are underlined in blue, green and red for neurotypical, patients with WS and Patients with ASD respectively. At the group level, the neurotypical decoding curve (blue) presents two marked peaks, one at $170 \mathrm{~ms}$ post stimulus onset, the second at $260 \mathrm{~ms}$ post stimulus onset. The ASD population (red curve) produced a significantly higher decoding peak than the WS population (green curve) at the earlier timing ( $170 \mathrm{~ms}-\mathrm{p}<0.05$ FWER corrected). A reversed pattern was found at the latter decoding peak (260ms post-stimulus onset, WS>ASD p<0.05 FWER corrected). On the right graph, the distribution of the peak for each individual in each group is plotted over time. The distribution in the neurotypical population (blue) and in patients with ASD (red) is bimodal with a dominance at 260ms for neurotypical participants and at $170 \mathrm{~ms}$ for patients with ASD. The distribution in WS patients is strictly unimodal.

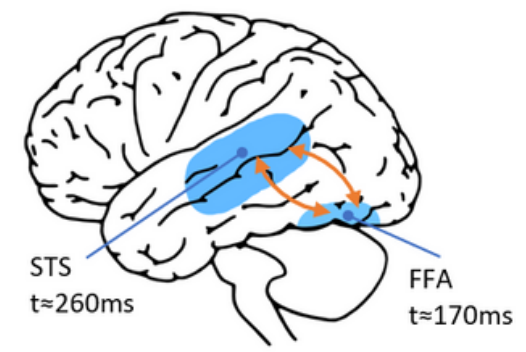

CTRL

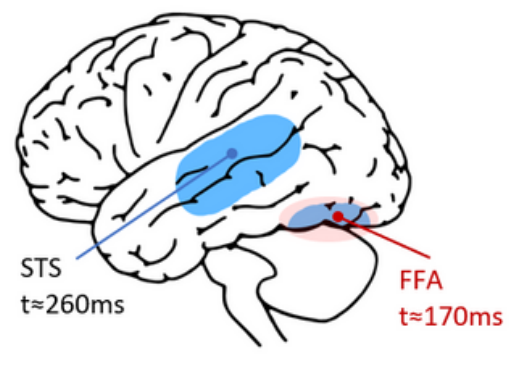

WS

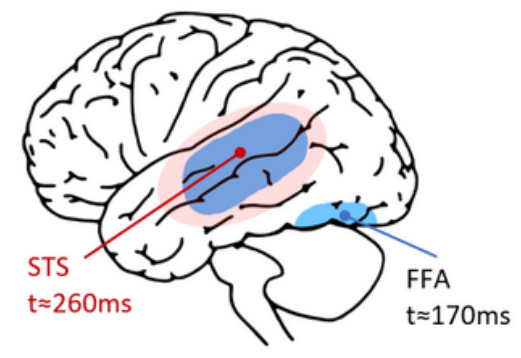

ASD

\section{Figure 4}

Time course of face processing along the temporo-occipital pathways in neurotypical subjects, WS and ASD. On the left, a schematic proposal of the two components interactions in face processing of neurotypical participants (blue): the early component (t=170ms) in the Fusiform face Area (FFA) and a late component ( $\mathrm{t}=260 \mathrm{~ms})$ in the Superior Temporal Sulcus (STS). In the middle, a schematic representation displaying the proposed altered functioning (in red) of the early component of face processing $(t=170 \mathrm{~ms})$ in patients with Williams syndrome (WS). On the right, a schematic representation displaying the proposed altered functioning (in red) of the late component $(\mathrm{t}=260 \mathrm{~ms})$ in patients with Autism Spectrum Disorder (ASD). 